\title{
Evaluation of Putty Metal for Internal Shielding for Patient Protection in Electron Therapy by Monte Carlo Study
}

\author{
Mohammad Javad Tahmasebi Birgani ${ }^{1,2}$, Mansour Zabihzadeh ${ }^{1}$, Saeideh Aliakbari ${ }^{1,{ }^{*}}$, Mohammad Ali \\ Behrouz $^{1}$ and Seyyed Mohammad Hosseini ${ }^{3}$ \\ ${ }^{1}$ Department of Medical Physics, School of Medicine, Ahvaz Jundishapur University of Medical Sciences, Ahvaz, Iran \\ ${ }^{2}$ Department of Radiotherapy and Radiation Oncology, Golestan Hospital, Ahvaz Jundishapur University of Medical Sciences, Ahvaz, Iran \\ ${ }^{3}$ Department of Radiation Therapy, Golestan Hospital, University of Jundishapur, Ahvaz, Iran \\ "Corresponding author: Assistant Professor, Department of Medical Physics, School of Medicine, Ahvaz Jundishapur University of Medical Sciences, Ahvaz, Iran. Tel: \\ +98-9354470112, Email: aliakbarisaeedeh@gmail.com
}

Received 2016 August 02; Revised 2017 May 05; Accepted 2017 August 17.

\begin{abstract}
Background: Lead has been widely used for internal shielding. It is recognized as a toxic material that pollutes the environment and does not fit well the patient's body because of its inflexibility.

Objectives: In this study, a specific combination (70\% W,18.61\% Ni, and 11.39\% C) of lead-free and flexible putty metal was introduced and validated for internal shielding by Monte Carlo study.

Methods: To evaluate the putty metal for internal shielding by Monte Carlo study, Varian 2100 C/D was validated within measurements. Then, using the given energy spectrum of Varian $2100 \mathrm{C} / \mathrm{D}$, the shield thickness and backscatter factors were calculated by Monte Carlo study and compared with those of lead.

Results: The results showed that this putty metal shield required a comparable thickness compared to lead for providing protection. In addition, it is nontoxic and flexible and can be easily cut. Internal shielding with high atomic number materials causes dose enhancement that is not taken into account in treatment planning systems. This study showed that this composition as an internal shield causes 5\% - 7\% reduction in electron backscatter factor compared to lead.

Conclusions: It can be concluded that lead can be replaced by putty metal with a specific combination (70\% $\mathrm{W}, 18.61 \% \mathrm{Ni}$, and $11.39 \%$ C). It is lead-free and flexible and its required thickness for protection is acceptable under clinical condition. It causes a 5\% - 7\% reduction in electron backscattering.
\end{abstract}

Keywords: Flexible Internal Shielding, Dose Enhancement, Electron Backscattering

\section{Background}

Shielding is used to protect underlying sensitive tissues in the treatment of superficial tumors using electron beams. It is most commonly applied when electromagnetic fields are used to treat the lip, buccal mucosa, and eyelid lesions (1).

Lead is widely used for internal shielding to protect non-target tissues in electron therapy $(2,3)$. Lead is recognized as a toxic element. It is not flexible enough to be easily shaped and thus, it would bring discomfort to the patient (4). Many studies have reported new substitute alloys to replace lead for internal or external shielding (5-8).

Many studies have investigated some new materials to decrease both shielding thickness and dose enhancement due to backscattering in electron therapy. Dia Chemical Co. developed a hard plate and a flexible shielding mate- rial called Rad-block and X-sheet, respectively. Rad-block is a hard plate while X-sheet provides flexible shielding; the latter is easily cut and fabricated. However, it has been reported that when these materials are used for internal shielding, backscattering might be a noticeable problem $(5,9)$.

When electrons pass through a target and meet a high atomic number material, they produce dose enhancement at the interface, as well as in the upstream direction. This dose enhancement is caused by electron backscattering from the interface. Many studies have measured or calculated the dose enhancement using Monte Carlo simulation (10-15).

To reduce dose enhancement, a material with a lower atomic number can be placed between the lead shield and the tissue, which is known as an absorber. The absorber creates less scatter than the higher atomic number shielding 
material. However, the absorber makes not only the shield thicker but also the prediction of the upstream dose difficult in treatment planning system (TPS) algorithms (16).

Because of the above reasons, in this study, a putty metal was investigated for internal shielding due to its lower electron backscattering, more flexibility, and nontoxic nature compared to lead. The acronym LFN was used to illustrate these three qualities.

\section{Objectives}

This study utilized a Monte Carlo simulation of a new kind of nontoxic material, which can be used for the protection against electron beams inside a human body.

\section{Methods}

\subsection{Chemical Analysis}

For simulating this material by MCNPX6 code, the elemental composition and the weight percentage of elements in LFN needed to be known. To determine the elements and weight percentage, energy-dispersive X-ray (EDX) spectroscopy and chemical analyses were run. The results of the EDX technique and chemical analysis are shown in Table 1.

\begin{tabular}{lc}
\hline \multicolumn{2}{l}{ Table 1. Elements and Weight Percentages of LFN Obtained by EDX Technique } \\
\hline Elements of LFN & Weight Percentage in LFN \\
\hline $\mathbf{W}$ & 70 \\
$\mathbf{C}$ & 11.39 \\
$\mathbf{N i}$ & 18.61 \\
\hline
\end{tabular}

The analysis of LFN by an electron microscope showed that the LFN was made of tungsten powder based on the polymer.

\subsection{Measurements}

To acquire the percentage depth dose (PDD) and dose profile, a $50 \times 50 \times 50 \mathrm{~cm}^{3}$ Scanditronix water phantom was set up. The PDD and dose profile were measured by a CC13 ionization chamber (volume $0.13 \mathrm{~cm}^{3}$, total active length $5.8 \mathrm{~mm}$, cylinder length $2.8 \mathrm{~mm}$, the inner diameter of cylinder $6.0 \mathrm{~mm}$, wall thickness $0.4 \mathrm{~mm}$, diameter of inner electrode $1.0 \mathrm{~mm}$, and length of inner electrode 3.3 $\mathrm{mm}$ ). The PDD and dose profile were measured and drawn by Omnipro-accept and Excel.

\subsection{Simulations}

In Monte Carlo simulations, MCNPX version 2.6.0. was used on a PC with a 3-GHz Intel Core Duo CPU running under Windows 7. Simulations were performed in the coupled electron-photon mode.

Varian $2100 \mathrm{C} / \mathrm{D}$ was modeled for 6 and $9 \mathrm{MeV}$ electron energies. The treatment head configuration consisted of a primary collimator, exit window, primary scattering foil, secondary scattering foil, monitor chamber, mirror, upper and lower jaws, and applicator. The manufacturer provided information about the geometry, material composition, and dimension of the components.

In this study, we assumed that the spectral distribution of the beam after the bending magnet (before electrons strike to the primary scattering foil) simulated an asymmetric Gaussian spectral distribution since the asymmetric Gaussian included unequal right FWHM (full-widthhalf-maximum) and left FWHM; thus, by using Equation 1, the spectral distribution of the initial electron was calculated, as follow (17):

$A\left(E, E_{p}, \sigma_{L}^{2}\right.$ OrR,$\left.r\right)$

$=\frac{2}{\sqrt{2 \pi}} \frac{1}{\sqrt{\sigma_{L O r R}^{2}(r+1)}}\left\{\begin{array}{c}\exp \left(-\frac{\left(E-E_{p}\right)^{2}}{2 \sigma_{R}^{2}}\right), E>E_{p} \\ \exp \left(-\frac{\left(E-E_{p}\right)^{2}}{2 \sigma_{L}^{2} r^{2}}\right), \text { Otherwise }\end{array}\right.$

where $\mathrm{E}_{\mathrm{p}}$ is the probable energy and $\mathrm{r}$ denotes the $\sigma_{L} / \sigma_{R}$ in which, $\sigma_{L}^{2}$ and $\sigma_{R}^{2}$ are left and right variances, respectively $\left(\sigma_{i}=2.335 F W H M_{i}\right)$.

Spatial spread or spot size was shown as the full width at half of the maximum (18). The parameters of the energy distribution for incident electrons are listed in Table 2 for each energy level. The number of particles was determined such that the statistical uncertainty would be within $\pm 1 \%$.

\begin{tabular}{lcc}
\hline Table 2. Parameters of Incident Electrons in Monte Carlo Simulations \\
\hline Variables & \multicolumn{2}{c}{ Values } \\
\hline Energy, MeV & 6 & 9 \\
\hline Spot Size, cm & 0.2 & 0.2 \\
\hline Mean energy, MeV & 6.05 & 9.02 \\
\hline Probable energy, MeV & 6.4 & 9.1 \\
\hline Right FWHM of the energy spectrum, MeV & 2.5 & 2.2 \\
\hline Left FWHM of the energy spectrum, MeV & 1.5 & 2 \\
\hline
\end{tabular}

Abbreviation: FWHM, full width half of maximum.

The calculated PDD and dose profile for two energies were verified against physical measurements within an accuracy of $3 \%$ and $3 \mathrm{~mm}$ for the $10 \times 10 \mathrm{~cm}^{2}$ applicator (Figure 1). The source-to-surface distance was $100 \mathrm{~cm}$. In this study, the differences between the calculated and measured values for 6 and $9 \mathrm{MeV}$ electron beams were analyzed by gamma index (19). 

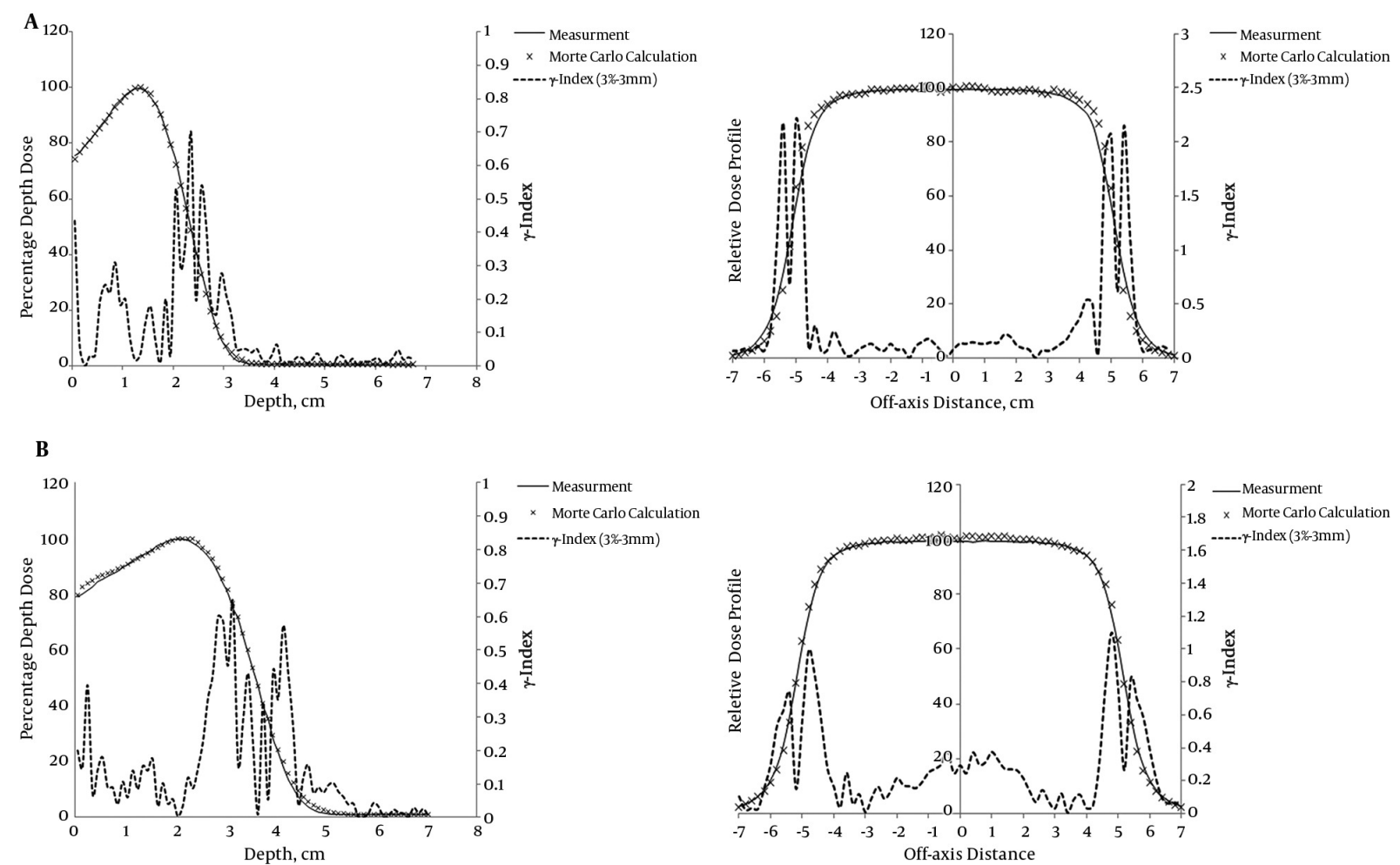

Figure 1. A, Calculated and measured $6 \mathrm{MeV}$ electron beam central axis depth dose distribution and beam profile of Varian $2100 \mathrm{C} / \mathrm{D}$ for a $10 \times 10 \mathrm{~cm}{ }^{2}$ applicator; B, calculated and measured $9 \mathrm{MeV}$ electron beam central axis depth dose distribution and beam profile of Varian $2100 \mathrm{C} / \mathrm{D}$ for a $10 \times 10 \mathrm{~cm}^{2}$ applicator

The internal shields were placed in a $25 \times 25 \times 25 \mathrm{~cm}^{3}$ water phantom and at two useful treatment depths for two energies. The dimensions of internal shields were $5 \times 5$ $\mathrm{cm}^{2}$ and three thicknesses were investigated.

First, the thickness of LFN was calculated at two depths intending to reduce the dose to $5 \%$ of the maximum dose of the open beam.

Second, the thickness of the lead shield was calculated at two depths for each energy level (minimum thickness (in millimeters) of lead required for blocking was given by electron energy in MeV divided by 2).

Third, the thickness of LFN was investigated with the same transmissions as the lead shied required.

Another parameter of internal shielding investigated was the backscatter factor (BSF, defined as the proportion of the dose at the shield interface with and without shielding) of each thickness of LFN that was determined and compared with that of lead.

\section{Results}

4.1. Chemical Analysis

Table 1 shows the result of the EDX technique and chemical analysis to find the elements and weight percentages of LFN.

\subsection{Parameters of Incident Electrons in Monte Carlo Simulation}

The parameters of the energy distribution of incident electrons are listed in Table 2 for energy levels of 6 and 9 $\mathrm{MeV}$ in the electron mode.

To compare the LFN with lead, many thicknesses were investigated to decrease transmission below $5 \%$ at the two most useful treatment depths for each energy level: 1.3 and $2 \mathrm{~cm}$ for $6 \mathrm{MeV}$ and 2 and $3 \mathrm{~cm}$ for $9 \mathrm{MeV}(1)$.

Subsequently, the required minimum thickness of lead was calculated at the two depths for each energy and finally, the thickness of LFN was investigated with the same transmission as the lead shied required. The thicknesses are shown in Tables 3 and 4.

The EBF of the required lead and LFN thicknesses were calculated by Monte Carlo simulations as shown in Tables 3 and 4. 
Table 3. The Thickness of Lead Required, the Thickness of LFN to Reduce the Dose to 5\% of the Maximum Dose of the Open Beam, the Thickness of LFN with the Same Transmission as Lead Required, and EBF at Two Depths in the Water Phantom Using the $6 \mathrm{MeV}$ Electron Beam

\begin{tabular}{|c|c|c|c|c|}
\hline \multirow{2}{*}{ Variables } & \multicolumn{2}{|c|}{ Depth $_{1}=1.3 \mathrm{~cm}$} & \multicolumn{2}{|c|}{ Depth $_{2}=2 \mathrm{~cm}$} \\
\hline & Thickness, mm & EBF & Thickness, mm & EBF \\
\hline Lead & 1.678 & 1.59 & 1 & 1.53 \\
\hline LFN (reduced transmission below $5 \%$ ) & 1.65 & 1.49 & 0.9 & 1.44 \\
\hline LFN (with the same transmission as lead) & 2.1 & 1.51 & 1.25 & 1.45 \\
\hline
\end{tabular}

Abbreviations: EBF, backscatter factor; LFN, putty metal (low backscattering - flexible - nontoxic)

Table 4. The Thickness of Lead Required, the Reasonable Thickness of LFN to Reduce the Dose to $5 \%$ of the Maximum Dose of the Open Beam, the Thickness of LFN with the Same Transmission as Lead Required, and EBF at Two Depths in the Water Phantom Using the 9 MeV Electron Beam

\begin{tabular}{|c|c|c|c|c|}
\hline \multirow{2}{*}{ Variables } & \multicolumn{2}{|c|}{ Depth $_{1}=2 \mathrm{~cm}$} & \multicolumn{2}{|c|}{ Depth $_{2}=3 \mathrm{~cm}$} \\
\hline & Thickness, mm & EBF & Thickness, mm & EBF \\
\hline Lead & 2.44 & 1.58 & 1.5 & 1.54 \\
\hline LFN (reduced transmission below 5\%) & 2.44 & 1.47 & 1.4 & 1.5 \\
\hline LFN (with the same transmission as lead) & 2.96 & 1.47 & 1.82 & 1.45 \\
\hline
\end{tabular}

Abbreviations: EBF, backscatter factor; LFN, putty metal (low backscattering - flexible - nontoxic)

\section{Discussion}

In order to validate the model employed for numerical simulations, the agreement between the simulated and measured PDD and beam profile should be provided.

As seen in Figure 1, the gamma index of PDD and dose profile are less than unity and within the accuracy of $3 \%$ and $3 \mathrm{~mm}$, except for the dose profile of 6 and $9 \mathrm{MeV}$. There is a sharp increase in the gamma index near the edge of the field size. These errors were out of the field size and did not make serious problems because, in this investigation, the dose on the central axis was more important. Using an ion chamber as a dosimeter and happening multiple scattering in low energies could be the reasons for sharp increases in gamma index curves in the dose profiles of 6 and $9 \mathrm{MeV}$, as seen in Figure 1. The error near the field edge for $9 \mathrm{MeV}$ is less than that of $6 \mathrm{MeV}$ that may be due to less multiple scattering at $9 \mathrm{MeV}$ compared to $6 \mathrm{MeV}(20,21)$.

In this study, another thickness of LFN was investigated, a thickness with the same transmission as the lead shield had. The results showed that the thickness of LFN was 1.2 times higher than that of lead, which still could be used in clinical conditions. This thickness of LFN is equal to the thickness reported by Lipowitz (22). The data of thicknesses are shown in Tables 3 and 4. Many studies have introduced different materials to replace lead and reported the thickness required for shielding. Tajiri et al. reported that the thickness required for shielding by Rad-block (hard plates) was slightly higher than that of lead, and the thickness of X-sheet (flexible shields) was 1.1 times higher than that required by lead (5).
The EBF values of LFN, shown in Tables 3 and 4, appear to be approximately $5 \%-7 \%$ less than those of lead are. It was shown that electron backscattering and dose enhancement of LFN were less than those of lead were, and it needed a thinner thickness of the absorber to reduce dose enhancement due to electron backscattering.

LFN, a putty metal with $70 \% \mathrm{~W}, 18.61 \% \mathrm{Ni}$, and $11.39 \% \mathrm{C}$ could be appropriate for internal shielding. It well fits the internal surfaces of the patient's body. Moreover, unlike lead shields, it has no toxic material in composition, which reduces environmental pollution. Furthermore, when LFN is used for internal shielding, electron backscattering is less when compared to lead; at the same time, the required thickness of LFN is acceptable clinically.

Internal shielding is used to protect underlying tissues in electron therapy. Lead is widely used for internal shielding but it is a toxic environmental pollutant. It is also inflexible and does not well fit the patient's body. This study investigated a lead-free and flexible material for internal shielding by Monte Carlo study.

Monte Carlo simulation played an important role in this study. The results of this study suggest a specific combination of $\mathrm{W}$ (70\%), C (11.39\%), and $\mathrm{Ni}(18.61 \%)$ for internal shielding in electron therapy. This material is flexible and much safer for the environment and the human body compared to lead. It well fits the internal surfaces of the patient's body and can be easily customized to arbitrary shapes. Backscattering due to shielding reduces compared to when the lead shield is used and at the same time, it needs a thinner absorber to reduce dose enhancement due to backscattering. From this study, it can be concluded that 
lead can be replaced by LFN for internal shielding.

\section{Acknowledgments}

The authors would like to thank the Radiotherapy and Oncology Center of Golestan Hospital of Jundishapur University of Medical Sciences for cooperation in the study and the vice chancellor for research of Jundishapur University of Medical Sciences for financial support.

\section{Footnotes}

Authors' Contribution: Study concept and design: Mohammad Javad Tahmasebi Birgani; acquisition of data: Mansour Zabihzadeh, Saeideh Aliakbari, and Mohammad Javad Tahmasebi Birgani; analysis and interpretation of data: Seyyed Mohammad Hosseini and Saeideh Aliakbari; drafting of the manuscript: Mohammad Ali Behrouz and Saeideh Aliakbari

Conflict of Interests: None declared.

Funding/Support: This study was financially supported by Jundishapur University of Medical Sciences, code number research project is U-93167.

\section{References}

1. Khan FM. The physics of radiation therapy. 4 th ed. Philadelphia: Lippincott Williams \& Wilkins; 2010.

2. Scuderi GJ, Brusovanik GV, Campbell DR, Henry RP, Kwon B, Vaccaro AR. Evaluation of non-lead-based protective radiological material in spinal surgery. Spine J. 2006;6(5):577-82. doi: 10.1016/j.spinee.2005.09.010. [PubMed: 16934731].

3. Ngaile JE, Uiso CB, Msaki P, Kazema R. Use of lead shields for radiation protection of superficial organs in patients undergoing head CT examinations. Radiat Prot Dosimetry. 2008;130(4):490-8. doi: 10.1093/rpd/ncn095. [PubMed: 18375945].

4. Nordberg GF, Fowler BA, Nordberg M. Handbook on the toxicology of metals. 4th ed. Cambridge, Massachusetts: Academic press; 2014

5. Tajiri M, Tokiya Y, Uenishi J, Sunaoka M, Watanabe K. New shielding materials for clinical electron beams. Radiother Oncol.2006;80(3):3913. doi: 10.1016/j.radonc.2006.08.002. [PubMed: 16959343].

6. Erdem M, Baykara O, Doğru M, Kuluöztürk F. A novel shielding material prepared from solid waste containing lead for gamma ray. Radiat Phys Chem. 2010;79(9):917-22. doi: 10.1016/j.radphyschem.2010.04.009.

7. Yue K, Yao Y, Dong X, Luo W. A novel shielding scheme studied by the Monte Carlo method for electron beam radiotherapy. Health Phys. 2013;104(3):277-81. doi: 10.1097/HP.ob013e31827a7d5f. [PubMed: 23361423].
8. Aghamiri MR, Mortazavi SMJ, Tayebi M, Mosleh-Shirazi MA, Baharvand $\mathrm{H}$, Tavakkoli-Golpayegani A, et al. A novel design for production of efficient flexible lead-free shields against X-ray photons in diagnostic energy range. J Biomed Phys Eng. 2011;1(1).

9. Perez-Calatayudt J, Ballester F, Serrano MA, Lluch JL, Casal E, Carmona V. Dosimetric characteristics of backscattered electrons in lead. Phys Med Biol. 2000;45(7):1841-9. [PubMed:10943922].

10. Chow IC, Grigorov GN. Monte Carlo simulation of backscatter from lead for clinical electron beams using EGSnrc. Med Phys. 2008;35(4):1241-50. doi: 10.1118/1.2874552. [PubMed: 18491516].

11. Shokrani P, Baradaran-Ghahfarokhi M, Zadeh MK. A novel approach in electron beam radiation therapy of lips carcinoma: A Monte Carlo study. Med Phys. 2013;40(4):41720. doi: 10.1118/1.4795756. [PubMed: 23556890].

12. Das IJ, Cheng CW, Mitra RK, Kassaee A, Tochner Z, Solin LJ. Transmission and dose perturbations with high-Z materials in clinical electron beams. Med Phys. 2004;31(12):3213-21. doi: 10.1118/1.1819551. [PubMed: 15651605].

13. Sathiyan S, Ravikumar M, Supe SS. Measurement of backscattered dose at metallic interfaces using high energy electron beams. Rep Pract Oncol Radiother. 2006;11(3):117-21. doi: 10.1016/s15071367(06)71056-2.

14. Heide B. Assessment of doses caused by electrons in thin layers of tissue-equivalent materials, using MCNP. Radiat Prot Dosimetry. 2013;156(4):495-505. doi: 10.1093/rpd/nct086. [PubMed: 23576794].

15. Kinhikar RA, Tambe CM, Patil K, Mandavkar M, Deshpande DD, Gujjalanavar R, et al. Estimation of dose enhancement to soft tissue due to backscatter radiation near metal interfaces during head and neck radiothearpy - A phantom dosimetric study with radiochromic film. J Med Phys. 2014;39(1):40-3. doi: 10.4103/0971-6203.125501. [PubMed: 24600171]. [PubMed Central: PMC3931227].

16. Seco J, Verhaegen F. Monte Carlo techniques in radiation therapy. Boca Raton, Florida: CRC press; 2013.

17. Kato T, Omachi S, Aso H. Asymmetric gaussian and its application to pattern recognition. In: Caelli T, Amin A, Duin RPW, de Ridder D, Kamel M, editors. Structural, syntactic, and statistical pattern recognition. SSPR/SPR 2002. 2396. Berlin, Heidelberg: Springer; 2002. p. 40513. doi: 10.1007/3-540-70659-3_42.

18. Pelowitz DB. MCNPXTM user's manual version 2. 6. 0. Los Alamos National Laboratory; 2007. 408 p.

19. Low DA, Harms WB, Mutic S, Purdy JA. A technique for the quantitative evaluation of dose distributions. Med Phys.1998;25(5):656-61. doi: 10.1118/1.598248. [PubMed: 9608475].

20. Jursinic PA, Nelms BE. A 2-D diode array and analysis software for verification of intensity modulated radiation therapy delivery. Med Phys. 2003;30(5):870-9. doi: 10.1118/1.1567831. [PubMed: 12772995].

21. Shi J, Simon WE, Zhu TC. Modeling the instantaneous dose rate dependence of radiation diode detectors. Med Phys. 2003;30(9):2509-19. doi: 10.1118/1.1602171. [PubMed: 14528973].

22. Khan FM, Doppke KP, Hogstrom KR, Kutcher GJ, Nath R, Prasad SC, et al. Clinical electron-beam dosimetry: Report of AAPM Radiation Therapy Committee Task Group No. 25. Med Phys.1991;18(1):73-109. doi: 10.1118/1.596695. [PubMed: 1901132]. 